\title{
Substratos alternativos ao xaxim no cultivo do híbrido Cattleya intermedia X Hadrolaelia purpurata (Orchidaceae)
}

\section{Alternative substrates for xaxim on culture of Cattleya intermedia $\mathrm{X}$ Hadrolaelia purpurata (Orchidaceae)}

\author{
Mauren Sorace ${ }^{1}$; Ricardo Tadeu de Faria ${ }^{2 *}$; Inês Cristina de Batista Fonseca ${ }^{3}$; \\ Lilian Yukari Yamamoto ${ }^{4}$; Maria Aparecida da Fonseca Sorace ${ }^{5}$
}

\begin{abstract}
Resumo
O presente trabalho teve como objetivo avaliar a eficiência de diferentes substratos de origem vegetal no cultivo de espécies de orquídeas. Plântulas de Cattleya intermedia X Hadrolaelia purpurata, provenientes de cultivo in vitro, aclimatizadas e com altura média de $5 \mathrm{~cm}$, foram plantadas nos substratos: 1-Xaxim desfibrado (testemunha); 2-Casca de arroz carbonizada; 3-Fibra de coco; 4-Casca de pínus; 5-Casca de pínus + Fibra de coco (1:1); 6-Casca de arroz + Fibra de coco (1:1); 7-Casca de arroz carbonizada + Casca de pínus (1:1); 8-Casca de pínus + Casca de arroz carbonizada + Fibra de coco (1:1:1). O delineamento experimental foi o inteiramente casualizado com oito tratamentos e dez repetições. Os dados obtidos foram submetidos à Análise de Variância e Teste de Tukey a 5\%. Os parâmetros de crescimento avaliados aos 17 meses foram: comprimento da raiz, número de raízes, altura da planta, número de brotos, número de folhas, comprimento da folha, largura da folha, massa fresca total e volume radicular. Os parâmetros dos substratos foram: potencial hidrogeniônico, condutividade elétrica e densidade. Conclui-se que todos os substratos testados podem ser recomendados para substituição do xaxim com destaque o substrato casca de arroz + casca de pínus, porém, o substrato constituído por fibra de coco foi o menos eficiente para o desenvolvimento vegetativo das plantas no cultivo do híbrido Cattleya intermedia $x$ Hadrolaelia purpurata.

Palavras-chave: Casca de arroz carbonizada, casca de pínus, fibra de coco, orquídeas
\end{abstract}

\begin{abstract}
The present work had the purpose of evaluating the efficiency of different substrates by vegetal origin, to grow species of orchids (Orchidaceae). Seedlings of Cattleya intermedia X Hadrolaelia purpurata, obtained by in vitro culture, and acclimated, with medium height of $5 \mathrm{~cm}$, had the following substrates: 1- Defibered xaxim (control); 2- Carbonized rice husk; 3- Coconut fiber; 4- Husk pínus; 5- Carbonized rice husk + Coconut fiber (1:1); 6- Carbonized rice husk + Husk pínus (1:1); 7- Coconut fiber + Husk pinus $(1: 1) ; 8$ - Husk pínus + Carbonized rice husk + Coconut fiber (1:1:1). A randomized design with eight treatments and ten replications. Results were submited to Variance Analysis and Tukey Test at 5\%. Growing parameters evaluated after 17 months were: root length, roots number, height of above ground, buds number, leaves number, leaf length, leaf width, total fresh mass and root volume.
\end{abstract}

1 Bióloga, Doutoranda em Agronomia. Universidade Estadual de Londrina (UEL); Bolsista da Capes. E-mail: mauren_band@ hotmail.com

2 Eng ${ }^{\circ}$ Agr., Dr. Professor Associado, Departamento de Agronomia (UEL). Londrina-Pr. E-mail: faria@uel.br

3 Eng ${ }^{a}$ Agr ${ }^{a}$, Dra. Professora Adjunta, Departamento de Agronomia (UEL). Londrina-Pr. E- mail: inescbf@uel.br

4 Eng $^{\circ} \mathrm{Agr}^{\mathrm{a}}$, Mestranda em Agronomia - Universidade Estadual de Londrina (UEL).

5 Eng ${ }^{a}$ Agr $^{\mathrm{a}}$, Dra. Professora da Universidade Estadual do Norte do Paraná (UENP) - Campus Luiz Meneghel. Bandeirantes-Pr. E-mail: sorace@ffalm.br

* Autor para correspondência 
Determinated substrates parameters were: $\mathrm{pH}$, eletric conductivity and density. All studied substrates can be recommended to substitute xaxim but, coconut fiber was least efficient to develop roots and aerial parts of plants. Better results were showed by substrates only with carbonized rice husk + husk pine on culture of Cattleya intermedia X Hadrolaelia purpurata.

Key words: Carbonized rice husk, husk pínus, coconut fiber, orchid

\section{Introdução}

A produção de orquídeas é uma atividade em crescente expansão nos mercados nacional e internacional, tornando-se evidente a necessidade de informações que permitam a otimização do seu cultivo (LORENZI; SOUZA, 2001).

Espécies de Cattleya e outros gêneros da família Orchidaceae são muito cultivadas no mundo inteiro como plantas ornamentais pela beleza de suas flores, o que thes confere grande importância econômica. Encontradas como nativas desde as regiões do México, América Central até a América do Sul compreendem cerca de 60 espécies e inúmeras variedades e híbridos.

Por muito tempo, para o cultivo em vasos, o xaxim ou samambaiaçu (Dicksonia sellowiana Hook.) foi o substrato mais utilizado pela maioria dos orquidófilos e produtores brasileiros. Por se tratar de espécie de crescimento muito lento, o xaxim deve ser preservado e o seu uso desestimulado. Em 1992, devido ao extrativismo desenfreado foi criada no Rio Grande do Sul, a lei 9.519 que proíbe a extração de xaxim em florestas nativas (GONÇALVES, 1992; KÄMPF, 2000; STRIGHETA et al., 2002). A exploração de D. sellowiana Hook. levou a sua inclusão em Listas Oficiais de Espécies da Flora Ameaçadas de Extinção (Portaria /IBAMA n'- 37-N/92 e COPAM 085/97) e no Apêndice II da Convenção Internacional sobre o Comércio Internacional de espécies da fauna e flora em perigo de extinção - CITES (INSTITUTO BRASILEIRO DO MEIO AMBIENTE E DOS RECURSOS NATURAIS - IBAMA, 2007), estudos vêm sendo realizados visando o uso de materiais alternativos ao xaxim.
De acordo com Backes e Kämpf (1991), no Brasil há vários materiais com potencial para exercer a função de substrato, entretanto, a falta de testes e informações limita sua exploração. No entanto, os resíduos produzidos na agricultura estão sendo utilizados no cultivo de plantas ornamentais proporcionando alternativas para o reaproveitamento de materiais com propriedades de biodegradação, antes descartados.

Segundo Kämpf (2000) e Souza (2003) os substratos utilizados no cultivo de orquídeas podem ser de origem vegetal, como turfa, esfagno, xaxim, caroço de açaí, fibra de coco, casca de arroz carbonizada, casca de pínus, nó de pinho, casca de peroba, carvão vegetal e piaçava; de origem mineral, como argila expandida, vermiculita, pedra brita, tijolo e até mesmo materiais de origem sintética, que servem como suporte às plantas, tais como poliestireno expansível (isopor) e espuma fenólica, além de complementar com uma camada de pedra brita, argila expandida ou cacos de vaso de argila no fundo para a drenagem da água. As características químicas e físicas dos substratos são muito importantes para o desenvolvimento das plantas e disponibilidade de nutrientes.

Apesar de existir uma grande diversidade de substratos e misturas a serem empregados no cultivo de orquídeas o seu sucesso depende da espécie e do tipo de ambiente onde será efetuado o cultivo (COOKE, 1999; RODRIGUES, 2001).

O objetivo deste trabalho foi avaliar a eficiência de substratos alternativos ao xaxim no cultivo de espécies de Cattleya (Orchidaceae). 


\section{Material e métodos}

O experimento foi conduzido no laboratório de Fitotecnia, Departamento de Agronomia, da Universidade Estadual de Londrina (UEL), localizada a $23^{\circ} 23^{\prime}$ de Latitude Sul, 51 ${ }^{\circ} 51^{\prime}$ de Longitude Oeste e altitude média de $556 \mathrm{~m}$, no período de maio de 2006 a novembro de 2007.

Foram utilizadas mudas do híbrido Cattleya intermedia X Hadrolaelia purpurata, com oito meses de idade, obtidas através de sementes germinadas in vitro, com aproximadamente $5 \pm$ $0,5 \mathrm{~cm}$ de altura. São plantas epífitas, apresentam crescimento simpodial, com duas folhas em cada pseudobulbo e duas flores em cada haste floral. O delineamento experimental utilizado foi inteiramente casualizado com oito tratamentos e 12 repetições.

Os tratamentos testados como substratos foram: T1 - Xaxim (testemunha); T2 - Casca de arroz carbonizada; T3 - Fibra de coco (Padrão 11Amafibra $\left.^{\circledR}\right)$; T4 - Casca de pínus; T5 - Casca de pínus + fibra de coco (Padrão 11-Amafibra $\left.{ }^{\circledR}\right)(1: 1$, v:v); T6 - Casca de arroz + fibra de coco (Padrão 11-Amafibra $\left.{ }^{\circledR}\right)(1: 1, \mathrm{v}: \mathrm{v}) ; \mathrm{T} 7$ - Casca de arroz + casca de pínus $(1: 1, \mathrm{v}: \mathrm{v})$; T8 - Pínus + arroz + coco (Padrão 11-Amafibra $\left.{ }^{\circledR}\right)(1: 1: 1, \mathrm{v}: \mathrm{v}: \mathrm{v})$.

As mudas foram colocadas em vasos de plástico transparente tipo 1 , com $8,5 \mathrm{~cm}$ diâmetro, $7,4 \mathrm{~cm}$ de altura e 4 furos na parte inferior, contendo uma camada de pedra brita número dois no fundo, para drenagem e a aeração do sistema radicular. Após o plantio, os vasos foram dispostos em mesas suspensas em casa de vegetação com $60 \%$ de sombreamento, com temperatura de $25^{\circ}-\mathrm{C}$, trocados aleatoriamente de posição a cada 15 dias a fim de eliminar possíveis efeitos de bordadura.

Após sete meses do início do experimento as mudas foram transplantadas para vasos de plástico de polipropileno de coloração preta, tipo 2 , com 10,5 $\mathrm{cm}$ de altura e $12,5 \mathrm{~cm}$ de diâmetro e com 4 furos na parte inferior e uma camada de pedra brita tipo 2, para drenagem e aeração do sistema radicular. Os vasos permaneceram sobre mesas suspensas, em casa de vegetação.

A cada trinta dias foram realizadas adubações foliares com a formulação NPK: 15-15-20, na concentração de $2 \mathrm{~g} \mathrm{~L}^{-1}$, sendo aplicados $25 \mathrm{~mL}$ dessa solução por vaso. A cada noventa dias efetuouse a adubação orgânica ( $\left.1 \mathrm{~g} \mathrm{vaso}^{-1}\right)$, composta de uma mistura de farinha de osso e torta de mamona, na proporção de 1:1 (SILVA, 1986). A irrigação foi realizada diariamente no verão e uma vez a cada dois dias no inverno, até capacidade do vaso.

Após 17 meses do início do experimento foram retiradas as mudas dos substratos e realizadas as avaliações dos seguintes parâmetros de crescimento: altura da parte aérea (APA), comprimento da maior raiz $(\mathrm{CMR})$, número de raízes $(\mathrm{NR})$, número de brotos (NB), número de folhas (NF), comprimento da maior folha (CMF), largura de folha (LF), massa fresca total (MFT), volume radicular (VR). Nos substratos foram avaliados o potencial hidrogeniônico $(\mathrm{pH})$, condutividade elétrica $(\mathrm{CE})$ e densidade (D).

A determinação do $\mathrm{pH}$ dos substratos foi efetuada através de peagâmetro. Para tanto, os vasos foram irrigados com água de $\mathrm{pH}$ conhecido $(6,5)$ até atingir a capacidade do campo. Após duas horas foram adicionados $100 \mathrm{~mL}$ de água por vaso, coletando-se o excedente em copos plásticos, para posterior medição do $\mathrm{pH}$ e condutividade elétrica (KÄMPF, 2000).

O volume radicular foi medido pelo deslocamento de água numa proveta de $1000 \mathrm{~mL}$ com a imersão do sistema radicular, coletando-se a água que transbordou da proveta e medindo-se o seu volume com uma proveta graduada de $100 \mathrm{~mL}$. Foram realizadas três leituras de cada repetição e tomou-se a média dessas medidas (BASSO, 2002).

Para determinar a condutividade elétrica dos tratamentos, foram colocados $25 \mathrm{~mL}$ da amostra de cada substrato adicionando-se $250(1: 10) \mathrm{mL}$ de água deionizada em um frasco de $500 \mathrm{~mL}$, agitandose por 30 minutos. Logo após, as amostras foram 
filtradas e as determinações feitas no extrato aquoso com o auxílio de um condutivímetro (SILVA; PORTO; KÄMPF, 1999).

A densidade do substrato foi medida utilizando um proveta de $500 \mathrm{~mL}$ preenchendo-se até a marca de $300 \mathrm{~mL}$ com o substrato na umidade atual. Em seguida, esta proveta é deixada cair, sob a ação de sua própria massa, de uma altura de $10 \mathrm{~cm}$, por 10 vezes consecutivas. Com o auxílio da espátula nivela-se a superfície levemente e lê-se o volume obtido (mL).

Os dados obtidos foram submetidos à Análise de Variância e Teste de Tukey à 5\%. Para as variáveis: número de raízes, número de folhas e número de brotos, os dados foram transformados em $\sqrt{x}_{\mathrm{x}}+1$, sendo que as médias apresentadas são as originais.

\section{Resultados e discussão}

Os efeitos dos substratos sobre o desenvolvimento de plantas da espécie do híbrido Cattleya intermedia X Hadrolaelia purpurata, após 17 meses, foram avaliados através dos parâmetros de crescimento: comprimento da maior raiz, número de raízes, volume radicular, altura da parte aérea, número de brotos, número de folhas, comprimento da maior folha, largura de folha e massa fresca total.

Na Tabela 1 são encontrados os resultados de comprimento da maior raiz, número de raízes e volume radicular, de plantas do híbrido Cattleya intermedia X Hadrolaelia purpurata.

Tabela 1. Médias referentes ao comprimento da maior raiz (CMR), número de raízes (NR) e volume radicular (VR) das plantas de Cattleya intermedia X Hadrolaelia purpurata, após 17 meses do início do experimento. Londrina, PR, 2007.

\begin{tabular}{lccc}
\hline \multicolumn{1}{c}{ Tratamentos } & $\begin{array}{c}\text { CMR } \\
(\mathbf{c m})\end{array}$ & $\begin{array}{c}\text { NR } \\
(*)\end{array}$ & $\begin{array}{c}\text { VR } \\
\left(\mathbf{m L}^{-1}\right)\end{array}$ \\
\hline T1-Xaxim & $44,60 \mathrm{a}^{* *}$ & $15,72 \mathrm{ab}$ & $21,16 \mathrm{a}$ \\
T2-Casca de arroz carbonizada & $27,22 \mathrm{~b}$ & $12,0 \mathrm{~b}$ & $15,91 \mathrm{~b}$ \\
T3-Fibra de coco & $29,96 \mathrm{~b}$ & $11,66 \mathrm{~b}$ & $15,41 \mathrm{~b}$ \\
T4-Casca de pínus & $27,38 \mathrm{~b}$ & $12,54 \mathrm{ab}$ & $19,25 \mathrm{ab}$ \\
T5-Casca de pínus + fibra de coco & $33,30 \mathrm{ab}$ & $17,41 \mathrm{ab}$ & $18,66 \mathrm{ab}$ \\
T6-Casca de arroz + fibra de coco & $28,88 \mathrm{~b}$ & $13,83 \mathrm{ab}$ & $15,16 \mathrm{~b}$ \\
T7-Casca de arroz + casca de pínus & $39,38 \mathrm{a}$ & $19,58 \mathrm{a}$ & $21,25 \mathrm{a}$ \\
T8-Pínus + arroz + coco & $24,68 \mathrm{~b}$ & $12,91 \mathrm{ab}$ & $16,50 \mathrm{~b}$ \\
\hline CV \% & $\mathbf{2 0 , 0 4}$ & $\mathbf{1 6 , 1 9}$ & $\mathbf{2 8 , 6 1}$ \\
\hline
\end{tabular}

*Dados sob transformação raiz quadrada $(\sqrt{ } \mathrm{x}+1)$.

**Médias seguidas de mesma letra na coluna não diferem entre si pelo Teste de Tukey a 5\% de significância.

Com base na análise estatística a média de comprimento da maior raiz do tratamento casca de arroz + casca de pínus não diferiu dos tratamentos com xaxim e casca de pínus + fibra de coco, e foi significativamente superior às obtidas nos demais substratos.

Para a variável número de raízes o substrato casca de arroz + casca de pínus, foi o que apresentou maior média, diferindo dos substratos com fibra de coco e com casca de arroz carbonizada.

Referente ao volume radicular, os tratamentos que apresentaram as maiores médias foram casca de arroz + casca de pínus e xaxim que diferiram, significativamente, dos substratos com fibra de coco e casca de arroz carbonizada. 
Quanto ao uso de fibra de coco como substrato, pode ser uma opção para substituir o xaxim em enraizamento de estacas de crisântemo segundo Bezerra et al. (2001), entretanto, tal como ocorreu com as espécies de Cattleya estudadas, não foi favorável ao desenvolvimento radicular de Dendrobium nobile cultivado com substrato de coco em cubos (ASSIS et al., 2005).

Yamakami et al. (2006), obtiveram resultados satisfatórios para volume radicular de Brassocattleya pastoral 'Rosa' e o híbrido Miltonidium cultivadas em substrato de casca de arroz carbonizada.
Os resultados obtidos para as três variáveis determinantes do desenvolvimento radicular demonstraram que os substratos casca de arroz + casca de pínus e o xaxim foram mais eficientes do que os substratos casca de arroz carbonizada, fibra de coco, casca de arroz + fibra de coco e pínus + arroz + coco. O substrato casca de pínus + fibra de coco pode ser uma opção por não diferir do xaxim.

Na Tabela 2 são encontrados os resultados da altura da parte aérea, número de brotos, número de folhas, comprimento da maior folha, largura de folha e massa fresca total, de plantas do híbrido Cattleya intermedia X Hadrolaelia purpurata.

Tabela 2. Médias referentes à altura da parte aérea (APA), número de brotos (NB), número folhas (NF), comprimento da maior folha (CMF), largura da folha (LF) e massa fresca total (MFT) das plantas de Cattleya intermedia X Hadrolaelia purpurata, após 17 meses do início do experimento. Londrina, PR, 2007.

\begin{tabular}{lcccccc}
\hline \multicolumn{1}{c}{ Tratamentos } & $\begin{array}{c}\text { APA } \\
(\mathbf{c m})\end{array}$ & $\begin{array}{c}\text { NB } \\
(*)\end{array}$ & $\begin{array}{c}\text { NF } \\
(*)\end{array}$ & $\begin{array}{c}\text { CMF } \\
(\mathbf{c m})\end{array}$ & $\begin{array}{c}\text { LF } \\
(\mathbf{c m})\end{array}$ & $\begin{array}{c}\text { MFT } \\
(\mathbf{m g})\end{array}$ \\
\hline T1-Xaxim & $25,87 \mathrm{a} * *$ & $5,25 \mathrm{a}$ & $7,83 \mathrm{a}$ & $17,75 \mathrm{a}$ & $3,03 \mathrm{a}$ & $62,54 \mathrm{a}$ \\
T2-Casca de arroz carbonizada & $21,72 \mathrm{ab}$ & $4,0 \mathrm{a}$ & $6,41 \mathrm{a}$ & $14,30 \mathrm{abc}$ & $2,68 \mathrm{ab}$ & $37,39 \mathrm{bc}$ \\
T3-Fibra de coco & $18,14 \mathrm{~b}$ & $3,91 \mathrm{a}$ & $6,16 \mathrm{a}$ & $11,72 \mathrm{c}$ & $2,30 \mathrm{~b}$ & $24,45 \mathrm{c}$ \\
T4-Casca de pínus & $23,31 \mathrm{ab}$ & $4,33 \mathrm{a}$ & $6,45 \mathrm{a}$ & $14,81 \mathrm{abc}$ & $2,81 \mathrm{ab}$ & $49,83 \mathrm{ab}$ \\
T5-Casca de pínus + fibra de coco & $24,26 \mathrm{ab}$ & $4,5 \mathrm{a}$ & $6,33 \mathrm{a}$ & $13,54 \mathrm{bc}$ & $3,11 \mathrm{a}$ & $44,76 \mathrm{~b}$ \\
T6-Casca de arroz + fibra de coco & $22,91 \mathrm{ab}$ & $4,5 \mathrm{a}$ & $6,66 \mathrm{a}$ & $15,04 \mathrm{abc}$ & $3,05 \mathrm{a}$ & $37,97 \mathrm{bc}$ \\
T7-Casca de arroz + casca de pínus & $27,33 \mathrm{a}$ & $4,83 \mathrm{a}$ & $7,5 \mathrm{a}$ & $17,51 \mathrm{a}$ & $3,21 \mathrm{a}$ & $51,12 \mathrm{ab}$ \\
T8-Pínus + arroz + coco & $24,25 \mathrm{ab}$ & $4,41 \mathrm{a}$ & $7,08 \mathrm{a}$ & $16,41 \mathrm{ab}$ & $2,79 \mathrm{ab}$ & $43,37 \mathrm{~b}$ \\
\hline CV \% & $\mathbf{2 1 , 4 3}$ & $\mathbf{1 4 , 3 6}$ & $\mathbf{1 1 , 1 5}$ & $\mathbf{2 0 , 9 6}$ & $\mathbf{1 7 , 4 5}$ & $\mathbf{2 6 , 7 7}$ \\
\hline
\end{tabular}

*Dados sob transformação raiz quadrada $(\sqrt{ } \mathrm{x}+1)$.

**Médias seguidas de mesma letra na coluna não diferem entre si pelo Teste de Tukey a 5\% de significância.

A análise das variáveis de crescimento avaliada demonstrou que os tratamentos xaxim e casca de arroz + casca de pínus não diferiram dos demais tratamentos com relação ao número de brotos e número de folhas e foram significativamente melhores para altura da parte aérea, comprimento da maior folha e massa fresca total.

Quanto aos substratos que foram prejudiciais ao crescimento das plantas, o tratamento com fibra de coco diferiu significativamente, do xaxim e casca de arroz + casca de pínus, para número de brotos e número de folhas que não variaram em função dos tratamentos. Substratos compostos por casca de arroz carbonizada e casca de arroz + fibra de coco reduziram a produção de massa fresca total e casca de pínus + fibra de coco afetou o crescimento longitudinal da maior folha diferindo significativamente, da testemunha (xaxim).

Com relação a altura da planta, foram obtidos resultados semelhantes com substrato fibra de coco 
com a espécie Brassocattleya pastoral 'Rosa' e com híbrido Miltonidium (YAMAKAMI, 2006). Também, não variaram em função dos substratos, os resultados de brotação de orquídeas, obtidos por Rego et al. (2000), Meneguce, Oliveira e Faria (2004) e Yamakami et al. (2006).

Quanto a massa fresca total, Yamakami et al. (2006), conseguiram resultados favoráveis de acúmulo de massa fresca com o substrato composto por casca de arroz carbonizada, contradizendo os resultados obtidos.

O potencial hidrogeniônico (pH), condutividade elétrica (CE) e densidade (D) dos substratos utilizados no cultivo do híbrido Cattleya intermedia X Hadrolaelia purpurata, após dezessete meses do início do experimento são apresentados na Tabela 3 .

Tabela 3. Médias referentes ao potencial hidrogeniônico (pH), condutividade elétrica (CE) e densidade (D) dos substratos utilizados no cultivo do híbrido Cattleya intermedia X Hadrolaelia purpurata, após 17 meses do início do experimento. Londrina, PR, 2007.

\begin{tabular}{lccc}
\hline \multicolumn{1}{c}{ Substratos } & $\mathbf{p H}$ & $\mathbf{C E}$ & $\mathbf{D}$ \\
$\left(\boldsymbol{\mu} \mathbf{S} \mathbf{~ c m}^{-\mathbf{1}}\right)$ & $\left(\mathbf{g ~ c m}^{-\mathbf{3}} \mathbf{)}\right.$ \\
\hline T1-Xaxim & $3,98 \mathrm{~d}$ & $202,48 \mathrm{a}$ & $0,44 \mathrm{a}$ \\
T2-Casca de arroz carbonizada & $5,65 \mathrm{a}$ & $234,52 \mathrm{a}$ & $0,41 \mathrm{a}$ \\
T3-Fibra de coco & $5,57 \mathrm{a}$ & $208,00 \mathrm{a}$ & $0,46 \mathrm{a}$ \\
T4-Casca de pínus & $3,70 \mathrm{e}$ & $222,72 \mathrm{a}$ & $0,45 \mathrm{a}$ \\
T5-Casca de pínus + fibra de coco & $4,08 \mathrm{~cd}$ & $233,10 \mathrm{a}$ & $0,45 \mathrm{a}$ \\
T6-Casca de arroz + fibra de coco & $5,45 \mathrm{a}$ & $210,20 \mathrm{a}$ & $0,44 \mathrm{a}$ \\
T7-Casca de arroz + casca de pínus & $4,20 \mathrm{c}$ & $239,26 \mathrm{a}$ & $0,42 \mathrm{a}$ \\
T8-Pínus + arroz + coco & $4,48 \mathrm{~b}$ & $254,24 \mathrm{a}$ & $0,46 \mathrm{a}$ \\
\hline CV \% & $\mathbf{2 , 5 6}$ & $\mathbf{2 6 , 5 6}$ & $\mathbf{1 6 , 6 4}$ \\
\hline
\end{tabular}

*Médias seguidas de mesma letra na coluna não diferem entre si pelo Teste de Tukey a $5 \%$ de significância.

Os valores de $\mathrm{pH}$ dos substratos utilizados variaram de 3,70 a 5,65 para $C$. intermedia X Hadrolaelia purpurata. Portanto, observou-se valores de $\mathrm{pH}$ mais baixos, inferiores a 4, para os substratos xaxim e composto por casca de pínus e valores mais altos, acima de 5, para os substratos com casca de arroz carbonizada e fibra de coco e casca de arroz + casca de pínus.

Segundo Gonçalves e Poggiani (1996) e Kämpf (2000), os valores ideais de $\mathrm{pH}$ de substratos para o cultivo de orquídeas epífitas, estão na faixa de 5,0 a 6,5 . O substrato que apresentou $\mathrm{pH}$ nesta faixa foi a casca de arroz + fibra de coco e os demais substratos apresentaram valores de $\mathrm{pH}$ inferiores a 5 .

Yamakami (2006), em cultivo de Miltonidium e do híbrido Brassocattleya pastoral 'Rosa' obteve para fibra de xaxim valores de $\mathrm{pH}$ de 4,5 e 4,8 respectivamente, que foram inferiores e diferiram estatisticamente, dos valores de $\mathrm{pH}$ dos substratos carvão + coco, casca de arroz carbonizada e casca de pínus + fibra de coco + casca de arroz carbonizada.

Os valores de $\mathrm{pH}$ obtidos para o substrato fibra de coco variaram entre 5,05 e 5,67, que foram considerados baixos, em comparação com substrato composto por pó de coco, que oscilou entre 6,3 e 6,7 (BEZERRA et al., 2001; LACERDA et al., 2006), e semelhantes aos obtidos por Yamamoto (2007), em cultivo de Miltonia regnelli Rchb.f. x Oncidium concolor Hook.

Segundo Yamakami et al. (2006), o substrato casca de arroz carbonizada apresentou valores de $\mathrm{pH}$ de 5,7 e 5,8 no cultivo de Brassocattleya 
pastoral 'Rosa' e Miltonidium respectivamente, que foram considerados levemente altos, mas não prejudicaram o desenvolvimento da planta.

Com relação ao $\mathrm{pH}$ de casca de pínus foram obtidos valores entre 3,67 e 3,70. Kämpf, Takane e Siqueira (2006) obtiveram valores entre 3,5 e 5,0. São considerados extremamente ácidos os valores abaixo de 4,5, que variam em função do tipo de pínus.

Em relação à condutividade elétrica e a densidade dos substratos pode-se observar que não houve diferença estatística entre os substratos testados para as espécies estudadas. Os valores de condutividade elétrica dos substratos utilizados variaram de 202,48 a $239,26 \mu \mathrm{S} \mathrm{cm}^{-1}$ (Tabela 3).

Wang e Gregg (1994) trabalharam com a variação de condutividade elétrica (CE) entre 63 a $380 \mu \mathrm{S} /$ $\mathrm{cm}$, em Phalaenopsis sp., entretanto não observaram diferenças no desenvolvimento vegetativo dessa orquídea.

Bernardi et al. (2004), no cultivo de Dendrobium nobile, realizando aplicações semanais com doses crescentes da solução nutritiva de Sarruge observaram ótimo desenvolvimento das plantas até a concentração de $125 \%$ sendo a melhor dose com $75 \%$, com uma condutividade elétrica de 144,6 $\mu \mathrm{S}$ $\mathrm{cm}^{-1}$. Para Yamakami et al. (2006) a condutividade elétrica variou de 73,3 a 98,3 $\mu \mathrm{S} \mathrm{cm}^{-1}$ para Brassocattleya pastoral 'Rosa' e Miltonidium.

Para densidade dos substratos, estas variaram de 0,41 a $0,46 \mathrm{~g} \mathrm{~cm}^{-3}$ para C. intermedia X Hadrolaelia purpurata (Tabela 3). No entanto, Bellé (1999) estudando substratos para o cultivo da espécie Maxillaria consanguinea var. pallida, encontrou valores de densidade em torno $0,315 \mathrm{~g} \mathrm{~cm}^{-3}$ para o substrato casca de pínus. Para casca de arroz carbonizada foi encontrado densidade em torno de $0,200 \mathrm{~g} \mathrm{~cm}^{-3}$ (KÄMPF; TAKANE; SIQUEIRA, 2006).

\section{Conclusões}

Conclui-se que o substrato fibra de coco foi o menos eficaz para o desenvolvimento radicular e vegetativo das plantas. Todos os demais substratos testados podem ser utilizados como alternativos ao xaxim, destacando-se o substrato casca de arroz carbonizada + casca de pínus.

\section{Referências}

ASSIS, A. M.; FARIA, R. T.; COLOMBO, L. A.; CARVALHO, J. F. R. P. Utilização de substratos a base de coco no cultivo de Dendrobium nobile Lindl. (Orchidaceae). Acta Scientiarum, Maringá, v. 27, n. 2, p. 255-260, 2005.

BACKES, M.A.; KÄMPF, AN. Substratos à base de composto de lixo urbano para a produção de plantas ornamentais. Pesquisa Agropecuária Brasileira, Brasília, v.26, n.5, p.753-758, 1991.

BASSO, F. M.; FARIA, R. T. Utilização de diferentes substratos no cultivo de Laelia lundii (Orchidaceae) visando a preservação do xaxim. In: MOSTRA ACADÊMICA DE TRABALHOS DE AGRONOMIA, 5., 2002, Londrina. Anais... Londrina, 2002. p. 48.

BELLÉ, S. Substituição da fibra de xaxim por casca de pínus no cultivo de Maxillaria consangüínea. In: ENCONTRO NACIONAL SOBRE SUBSTRATOS PARA PLANTAS, 1., 1999, Porto Alegre. Anais... Porto Alegre, 1999. p.183-189.

BERNARDI, A. C.; FARIA, R. T.; CARVALHO, J. R. P.; UNEMOTO, K. L.; ASSIS, A. M. Desenvolvimento vegetativo de plantas de Dendrobium nobile Lindl. fertirrigadas com diferentes concentrações de solução nutritiva de Sarruge. Semina: Ciências Agrárias, Londrina, v. 25, n. 1, p. 11-18, 2004.

BEZERRA, F. C.; ROSA, M. F.; BRÍGIDO, A. K. L.; NORÕES, E. R. V. Utilização de pó de coco como substrato de enraizamento para estacas de crisântemo. Revista Brasileira de Horticultura Ornamental, Campinas, v. 7, n. 2, p. 129-134, 2001.

COOKE, R. B. Estufas e telados. Revista Oficial da Orquidário, Rio de Janeiro, v. 13, n. 3/4, p. 94-101, 1999.

GONÇALVES, A. L. Características de substratos. In: CASTRO, C. E. F.; ANGELIS, B. L. D.; MOURA, L. P. P.; SILVEIRA, R. B. A.; ANGELIS NETO, G.;SATO, N. T. Manual de Floricultura. Maringá: SBFPO, 1992. p. 44-52. 
GONÇALVES, J. L. M.; POGGIANI, F. Substratos para a produção de mudas florestais. In: CONGRESSO LATINO AMERICANO DE CIÊNCIA DE SOLO, 13., Águas de Lindóia, 1996. Resumos... Piracicaba: Sociedade Latino Americana de Ciência do Solo, 1996. CD-ROM.

INSTITUTO BRASILEIRO DO MEIO AMBIENTE E DOS RECURSOS NATURAIS - IBAMA. Convenção sobre o Comércio Internacional de Espécies da Flora e Fauna Selvagens em Perigo de Extinção. Disponível em: $<$ http://www.ibama.gov.br/>. Acesso em: 6 ago. 2007.

KÄMPF, A. N. Produção comercial de plantas ornamentais. Porto Alegre: Agropecuária, 2000.

KÄMPF, A. N.; TAKANE, R. J.; SIQUEIRA, P. T. V. Floricultura: técnicas de preparo de substratos. Brasília: LK Editora e Comunicação, 2006. 132 p.

LACERDA, M. R. B.; PASSOS, M. A. A.; RODRIGUES, J. J. V.; BARRETO, L. P. Características físicas e químicas de substratos a base de pó de coco e resíduo de sisal para produção de mudas de sabiá (Mimosa caesalpiniaefolia Benth). Revista Árvore, Viçosa, v. 30, n. 2, p. 163-170, 2006.

LORENZI, H. S.; SOUZA, H. M. Plantas Ornamentais no Brasil. 3. ed. Nova Odessa: Ed Plantarum Ltda, 2001. 835 p. 3 v.

MENEGUCE, B.; OLIVEIRA, R. B. D.; FARIA, R. T. Propagação vegetativa de Epidendrum ibaguense Lindl. (Orchidaceae) em substratos alternativos ao xaxim. Semina: Ciências Agrárias, Londrina, v. 25, n. 2, p. 101106, 2004.

REGO, L. V.; BERNARD, A.; TAKAHASHI, L. S. A.; FARIA, R. T. Desenvolvimento vegetativo de genótipos de orquídeas brasileiras em substratos alternativos ao xaxim. Revista Brasileira de Horticultura Ornamental, Campinas, v. 6, n. 1, p. 75-79, 2000.
RODRIGUES, V. T. Substratos e cultivo. Boletim da Coordenadoria das Associações Orquidófilas do Brasil (CAOB), Rio de Janeiro, n. 44, p. 50-54, 2001.

SILVA, W. Cultivo de orquídeas no Brasil. São Paulo: Nobel, 1986.96 p.

SILVA, L.; PORTO, M. D. M.; KÄMPF, A. N. Características químicas e físicas de substratos à base de turfa e casca de arroz carbonizada. In: ENCONTRO NACIONAL SOBRE SUBSTRATOS PARA PLANTAS (ENSUB), 1., 1999, Porto Alegre. Anais... Porto Alegre, 1999, p. 235-240.

SOUZA, M. Muito além do xaxim. Natureza, São Paulo, v. 182, n. 2, p. 32-37, 2003.

STRIGHETA, A. C. O.; LIRIO, V. S.; SILVA, C. A. B.; REIS, B. S.; AGUIAR, D. R. D. Diagnóstico do segmento de produção da cadeia produtiva de flores e plantas ornamentais do Rio de Janeiro. Revista Brasileira de Horticultura Ornamental, Campinas, v. 8, n. 1/2, p.7790, 2002.

WANG, Y. T.; GREGG, L. L. Médium and fertilizer affect the performance of Phalaenopsis during two flowers cycles. HortScience, Alexandria, v. 29, n. 4, p. 269-270, 1994.

YAMAMOTO, Y. Y. Substratos a base de coco de bagaço de cana-de-açúcar no cultivo de orquídea. 2007. TCC (Trabalho de Conclusão de Curso) - Universidade Estadual de Londrina, Londrina, 2007.

YAMAKAMI, J. K. Uso de resíduos agrícolas como substrato alternativo ao xaxim no cultivo de orquídeas. 2006. Dissertação (Mestrado em Fitotecnia) Universidade Estadual de Londrina, Londrina, 2006.

YAMAKAMI, J. K.; FARIA, R. T.; ASSIS, A. M.; REGO, L. V. Cultivo de Cattleya Lindley (Orchidaceae) em substratos alternativos ao xaxim. Acta Scientiarum, Maringá, v. 28, n. 4, p. 523-526, 2006. 